\title{
ANÁLISE DA PRODUÇÃO CIENTÍFICA EM GERIATRIA E GERONTOLOGIA
}

Guilherme Briczinski de Souza; Universidade Federal de Ciências da Saúde de Porto Alegre; gbriczinski@gmail.com

Fernanda Górski; Universidade Federal de Ciências da Saúde de Porto Alegre;fergorski@gmail.com Juliane Pinto Lucero; Universidade Federal de Ciências da Saúde de Porto Alegre; julianeplucero@gmail.com Julyhe Nunes Paulin; Universidade Federal de Ciências da Saúde de Porto Alegre; paulinjulyhe@gmail.com

Eduardo Garcia; Universidade Federal de Ciências da Saúde de Porto Alegre; eduardosgarcia@terra.com

\section{RESUMO}

Introdução: A geriatria e a gerontologia são áreas que trabalham na promoção, prevenção, tratamento e reabilitação da saúde do idoso. Sabe-se que os idosos-estão sujeitos à diversas manifestações patológicas, sendo compreensível que ocorra um alto número de pesquisas sobre a saúde dos mesmos. Objetivo: Analisar a produção científica publicada nos anais da Jornada de Inverno da Sociedade Brasileira de Geriatria e Gerontologia (SBGG). Metodologia: Revisão integrativa, no qual foram analisados anais de 2017 a 2019 da Jornada de Inverno da SBGG disponíveis na internet. Foi construído um banco de dados contendo dados referentes à quantidade de resumos por ano, tipo de estudo, sexo, estado e profissão do autor. Para a análise dos dados foi criada tabela de contingência. Resultados: Foram 185 trabalhos, sendo que em 2017 houve maior produção científica 44,8\%. Dentre os tipos de estudos 64,9\% foram estudos transversais, e $13 \%$ de revisão de literatura. Houve a predominância de autoras $(78,9 \%)$ em todas as três edições. Quanto ao estado brasileiro com mais publicações está o Rio Grande do Sul com 74,5\%, Santa Catarina 9,8\%, Paraná 6,5\% e São Paulo 3,3\%. Contudo, notamos que a maioria dos autores são da Enfermagem e Medicina com $20 \%$ cada, seguido da Nutrição 17,8\% e Fisioterapia 16,2\%. Conclusão: A jornada contribuiu para a divulgação multidisciplinar da saúde do idoso, entretanto falta o incremento de áreas como a fonoaudiologia, terapia ocupacional, odontologia para aumentar os estudos nessa área.

Palavras-chave: Congressos como Assunto; Gerontologia; Pesquisa. 